\title{
Singularity of the Magnetic-Field Integral Equation and its Extraction
}

\author{
Levent Gürel, Senior Member, IEEE, and Özgür Ergül, Student Member, IEEE
}

\begin{abstract}
In the solution of the magnetic-field integral equation (MFIE) by the method of moments (MOM) on planar triangulations, singularities arise both in the inner integrals on the basis functions and also in the outer integrals on the testing functions. A singularity-extraction method is introduced for the efficient and accurate computation of the outer integrals, similar to the way inner-integral singularities are handled. In addition, various formulations of the MFIE and the electric-field integral equation are compared, along with their associated restrictions.
\end{abstract}

Index Terms-Electric-field integral equation, integral equations, magnetic-field integral equation, moment methods, numerical analysis, singularity extraction.

\section{INTRODUCTION}

$\mathbf{I}$ $\mathrm{N}$ THIS LETTER, we introduce a novel and efficient technique to accurately compute the highly singular integrals encountered in the method of moments (MOM) solution of the magnetic-field integral equation (MFIE) discretized on planar triangulations. This is achieved by applying a new singularityextraction method to the outer integrals of the MFIE [1], in addition to the well-known singularity-extraction operation performed on the inner integrals [2], [3].

For conducting scatterers with closed surfaces, the MFIE can be written directly from the boundary condition for the tangential magnetic field on the surface as

$$
\boldsymbol{J}(\boldsymbol{r})-\hat{\boldsymbol{n}} \times \int_{S} d \boldsymbol{r}^{\prime} \boldsymbol{J}\left(\boldsymbol{r}^{\prime}\right) \times \nabla^{\prime} g\left(\boldsymbol{r}, \boldsymbol{r}^{\prime}\right)=\hat{\boldsymbol{n}} \times \boldsymbol{H}^{i}(\boldsymbol{r})
$$

by simply expressing the scattered magnetic field in terms of the induced (unknown) surface current $\boldsymbol{J}(\boldsymbol{r})$. In (1), the observation point $\boldsymbol{r}$ approaches the surface from the outside, $\hat{\boldsymbol{n}}$ is the outwardly directed normal, $\boldsymbol{H}^{\boldsymbol{i}}(\boldsymbol{r})$ is the incident magnetic field, and

$$
g\left(\boldsymbol{r}, \boldsymbol{r}^{\prime}\right)=\frac{e^{i k R}}{4 \pi R} \quad\left(R=\left|\boldsymbol{r}-\boldsymbol{r}^{\prime}\right|\right)
$$

denotes the free-space Green's function in phasor notation with the $e^{-i w t}$ convention.

Manuscript received October 26, 2004; revised May 5, 2005. This work was supported by the Turkish Academy of Sciences in the framework of the Young Scientist Award Program (LG/TUBA-GEBIP/2002-1-12), by the Scientific and Technical Research Council of Turkey (TUBITAK) under Research Grant 103E008, and by contracts from ASELSAN and SSM.

The authors are with the Department of Electrical and Electronics Engineering, Bilkent University, TR-06800 Ankara, Turkey (e-mail: lgurel@bilkent.edu.tr; ergul@ee.bilkent.edu.tr).

Digital Object Identifier 10.1109/LAWP.2005.851103
Solution of (1) with the MOM requires the expansion of $\boldsymbol{J}(\boldsymbol{r})$ in a series of basis functions (BFs) $\boldsymbol{b}_{n}(\boldsymbol{r})$, i.e.,

$$
\boldsymbol{J}(\boldsymbol{r})=\sum_{n=1}^{N} a_{n} \boldsymbol{b}_{n}(\boldsymbol{r})
$$

and projection of the boundary condition (1) onto $N$ testing functions (TFs) $\boldsymbol{t}_{m}(\boldsymbol{r})$. Then, the $N \times N$ matrix equation is obtained as

$$
\sum_{n=1}^{N} Z_{m n}^{M} a_{n}=v_{m}^{M}, \quad m=1, \ldots, N
$$

where $a_{n}$ is the unknown coefficient of the $n$th BF. Matrix elements $Z_{m n}^{M}$ can be derived as

$$
\begin{aligned}
Z_{m n}^{M}= & \int_{S_{m}} d \boldsymbol{r} \boldsymbol{t}_{m}(\boldsymbol{r}) \cdot \boldsymbol{b}_{n}(\boldsymbol{r}) \\
& -\int_{S_{m}} d \boldsymbol{r} \boldsymbol{t}_{m}(\boldsymbol{r}) \cdot \hat{\boldsymbol{n}} \times \int_{S_{n}} d \boldsymbol{r}^{\prime} \boldsymbol{b}_{n}\left(\boldsymbol{r}^{\prime}\right) \times \nabla^{\prime} g\left(\boldsymbol{r}, \boldsymbol{r}^{\prime}\right) .
\end{aligned}
$$

Similar to the MFIE, the electric-field integral equation (EFIE) is based on the boundary condition for the tangential electric field on the surface, i.e.,

$$
\hat{\boldsymbol{t}} \cdot \int_{S} d \boldsymbol{r}^{\prime} \boldsymbol{J}\left(\boldsymbol{r}^{\prime}\right) \cdot\left(\overline{\mathbf{I}}-\frac{\nabla \nabla^{\prime}}{k^{2}}\right) g\left(\boldsymbol{r}, \boldsymbol{r}^{\prime}\right)=\frac{i}{k \eta} \hat{\boldsymbol{t}} \cdot \mathbf{E}^{i}(\boldsymbol{r})
$$

where $\hat{\boldsymbol{t}}$ denotes any tangential direction at any point $\boldsymbol{r}$ on the surface. In (1) and (6), $\boldsymbol{J}(\boldsymbol{r})$ denotes the same induced surface current density, which can be expanded using (3). Discretization of (6) is completed with the use of the TFs $\boldsymbol{t}_{m}(\boldsymbol{r})$, to obtain

$$
\sum_{n=1}^{N} Z_{m n}^{E} a_{n}=v_{m}^{E}, \quad m=1, \ldots, N
$$

where

$$
\begin{aligned}
Z_{m n}^{E} & =\int_{S_{m}} d \boldsymbol{r} \boldsymbol{t}_{m}(\boldsymbol{r}) \cdot \int_{S_{n}} d \boldsymbol{r}^{\prime} \boldsymbol{b}_{n}\left(\boldsymbol{r}^{\prime}\right) g\left(\boldsymbol{r}, \boldsymbol{r}^{\prime}\right) \\
& -\frac{1}{k^{2}} \int_{S_{m}} d \boldsymbol{r} \boldsymbol{t}_{m}(\boldsymbol{r}) \cdot \int_{S_{n}} d \boldsymbol{r}^{\prime} \boldsymbol{b}_{n}\left(\boldsymbol{r}^{\prime}\right) \cdot\left[\nabla \nabla^{\prime} g\left(\boldsymbol{r}, \boldsymbol{r}^{\prime}\right)\right] .
\end{aligned}
$$

Comparing (6) to (1) and (8) to (5), EFIE is clearly more singular than MFIE due to the double derivative operators. In order to facilitate the computation of (8), it is a common practice [4] to distribute these two differential operators onto the basis and testing functions (BTFs) to arrive at

$$
\begin{aligned}
& Z_{m n}^{E}=\int_{S_{m}} d \boldsymbol{r} \boldsymbol{t}_{m}(\boldsymbol{r}) \cdot \int_{S_{n}} d \boldsymbol{r}^{\prime} g\left(\boldsymbol{r}, \boldsymbol{r}^{\prime}\right) \boldsymbol{b}_{n}\left(\boldsymbol{r}^{\prime}\right) \\
& \quad-\frac{1}{k^{2}} \int_{S_{m}} d \boldsymbol{r}\left[\nabla \cdot \boldsymbol{t}_{m}(\boldsymbol{r})\right] \int_{S_{n}} d \boldsymbol{r}^{\prime} g\left(\boldsymbol{r}, \boldsymbol{r}^{\prime}\right)\left[\nabla^{\prime} \cdot \boldsymbol{b}_{n}\left(\boldsymbol{r}^{\prime}\right)\right]
\end{aligned}
$$


which is less singular than the MFIE matrix element (5). This reduction in the degree of the singularity in EFIE can be obtained at the cost of placing restrictions on the BTFs. That is, $\boldsymbol{b}_{n}(\boldsymbol{r})$ and $\boldsymbol{t}_{m}(\boldsymbol{r})$ in (9) are restricted to be divergence-conforming functions (whose divergences are defined and nonsingular), whereas no such restriction is necessary in (8).

\section{MFIE FORMULATIONS}

Taking a hint from the conversion of (8) to (9) for the EFIE, we can move the differential operator in (5) from the Green's function onto either the BF or the TF. By using standard vector identities and the

$$
\nabla^{\prime}=\nabla_{S}^{\prime}+\hat{n}^{\prime} \frac{\partial}{\partial n^{\prime}}
$$

decomposition, we can manipulate (5) to obtain a second formulation as [5]

$$
\begin{aligned}
Z_{m n}^{M}= & \int_{S_{m}} d \boldsymbol{r} \boldsymbol{t}_{m}(\boldsymbol{r}) \cdot \hat{\boldsymbol{n}} \times \int_{S_{n}} d \boldsymbol{r}^{\prime} \nabla_{S}^{\prime} \times\left[g\left(\boldsymbol{r}, \boldsymbol{r}^{\prime}\right) \boldsymbol{b}_{n}\left(\boldsymbol{r}^{\prime}\right)\right] \\
& -\int_{S_{m}} d \boldsymbol{r} \boldsymbol{t}_{m}(\boldsymbol{r}) \cdot \hat{\boldsymbol{n}} \times \int_{S_{n}} d \boldsymbol{r}^{\prime} g\left(\boldsymbol{r}, \boldsymbol{r}^{\prime}\right) \nabla_{S}^{\prime} \times \boldsymbol{b}_{n}\left(\boldsymbol{r}^{\prime}\right) \\
& -\int_{S_{m}} d \boldsymbol{r} \boldsymbol{t}_{m}(\boldsymbol{r}) \cdot \hat{\boldsymbol{n}} \times \int_{S_{n}} d \boldsymbol{r}^{\prime} \boldsymbol{b}_{n}\left(\boldsymbol{r}^{\prime}\right) \times \hat{\boldsymbol{n}}^{\prime} \frac{\partial g\left(\boldsymbol{r}, \boldsymbol{r}^{\prime}\right)}{\partial n^{\prime}} \\
& +\int_{S_{m}} d \boldsymbol{r} \boldsymbol{t}_{m}(\boldsymbol{r}) \cdot \boldsymbol{b}_{n}(\boldsymbol{r})
\end{aligned}
$$

where the $\nabla_{S}^{\prime}$ operator is transferred onto the BF. Therefore, in this second formulation the BF is restricted to be a curl-conforming function, however, no restriction exists on the TF. Alternatively, (5) can be manipulated to obtain a third formulation as

$$
\begin{aligned}
Z_{m n}^{M}= & \int_{S_{m}} d \boldsymbol{r} \nabla_{S} \cdot\left\{\left[\boldsymbol{t}_{m}(\boldsymbol{r}) \times \hat{\boldsymbol{n}}\right] \times \int_{S_{n}} d \boldsymbol{r}^{\prime} \boldsymbol{b}_{n}\left(\boldsymbol{r}^{\prime}\right) g\left(\boldsymbol{r}, \boldsymbol{r}^{\prime}\right)\right\} \\
& -\int_{S_{m}} d \boldsymbol{r}\left\{\nabla_{S} \times\left[\boldsymbol{t}_{m}(\boldsymbol{r}) \times \hat{\boldsymbol{n}}\right]\right\} \cdot \int_{S_{n}} d \boldsymbol{r}^{\prime} \boldsymbol{b}_{n}\left(\boldsymbol{r}^{\prime}\right) g\left(\boldsymbol{r}, \boldsymbol{r}^{\prime}\right) \\
& -\int_{S_{m}} d \boldsymbol{r} \boldsymbol{t}_{m}(\boldsymbol{r}) \cdot \hat{\boldsymbol{n}} \times \hat{\boldsymbol{n}} \times \int_{S_{n}} d \boldsymbol{r}^{\prime} \boldsymbol{b}_{n}\left(\boldsymbol{r}^{\prime}\right) \frac{\partial g\left(\boldsymbol{r}, \boldsymbol{r}^{\prime}\right)}{\partial n} \\
& +\int_{S_{m}} d \boldsymbol{r} \boldsymbol{t}_{m}(\boldsymbol{r}) \cdot \boldsymbol{b}_{n}(\boldsymbol{r})
\end{aligned}
$$

where the derivative operator is applied to the TF. In this third formulation, there is no restriction on the $\mathrm{BF}$, however, the TF is restricted to be a divergence-conforming function since

$$
\nabla_{S} \times\left[\boldsymbol{t}_{m}(\boldsymbol{r}) \times \hat{\boldsymbol{n}}\right]=-\hat{\boldsymbol{n}} \nabla_{S} \cdot \boldsymbol{t}_{m}(\boldsymbol{r}) .
$$

Since the first formulation given by (5), which is widely used in the literature [6]-[10], does not place any restrictions on either the BF or the TF, it can be used with both divergence-conforming and curl-conforming functions.

\section{SINGULARITY OF THE MFIE}

For both EFIE and MFIE, the order of singularity arising in computations depends on the formulation. Despite the availability of different formulations together with various choices

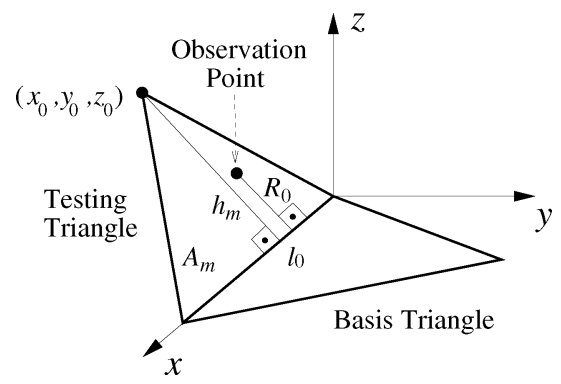

Fig. 1. Generic configuration of two neighboring triangles.

of BTFs, using (5) for the MFIE [6]-[10] and (9) for the EFIE with the Rao-Wilton-Glisson (RWG) BTFs [4] on planar triangulations appears to be the most popular in the literature. Both (5) and (9) have singular inner integrals, which can be accurately computed by employing the well-known singularity-extraction techniques [2], [3]. However, since the inner integral of (5) is more singular than that of (9), the outer integral of the MFIE contains a logarithmic singularity, which is not encountered in the EFIE. ${ }^{1}$ Since the logarithmic singularity is quite mild, it may be possible to compute the outer integral of the MFIE to the desired accuracy by sampling the integral inside the testing triangle with a sufficiently high number of integration points. Nevertheless, we propose a more efficient way to handle this singularity in order to obtain the same accuracy with fewer number of integration points.

The singularity in the outer integral of the MFIE is observed for the interactions between the neighboring triangles and does not cause problems if the testing and basis triangles are on the same plane. However, if the neighboring triangles are on different planes as shown in Fig. 1, the singularity becomes significant since the singular field on the common edge is required to be tested. In that case, the computations clearly benefit from the improved singularity-extraction technique presented in this paper. For example, the configuration depicted in Fig. 1 involves interactions between the two triangles that contain such problematic integrals (in the imaginary part of the matrix element) as

$$
I=\int_{S_{m}} d \boldsymbol{r}\left(\begin{array}{c}
1 \\
x \\
x^{2}
\end{array}\right) \int_{S_{n}} d \boldsymbol{r}^{\prime} \frac{\left(y-y^{\prime}\right)}{R^{3}}
$$

where the inner integral evaluates to a logarithmic function. In order to extract the logarithmic singularity, (14) can be rearranged as

$$
\begin{array}{r}
I=\int_{S_{m}} d \boldsymbol{r}\left(\begin{array}{c}
1 \\
x \\
x^{2}
\end{array}\right)\left\{\int_{S_{n}} d \boldsymbol{r}^{\prime} \frac{\left(y-y^{\prime}\right)}{R^{3}}+2 \ln \left(R_{0}\right)\right\} \\
-\int_{S_{m}} d \boldsymbol{r}\left(\begin{array}{c}
1 \\
x \\
x^{2}
\end{array}\right) 2 \ln \left(R_{0}\right)
\end{array}
$$

\footnotetext{
${ }^{1}$ Indeed, outer integrals of the EFIE contain some $R_{0} \ln \left(R_{0}\right)$ terms, where $R_{0}$ is the perpendicular distance from the observation point to the edge of the basis triangle. However, these logarithmic terms are not singular as $R_{0} \rightarrow 0$.
} 


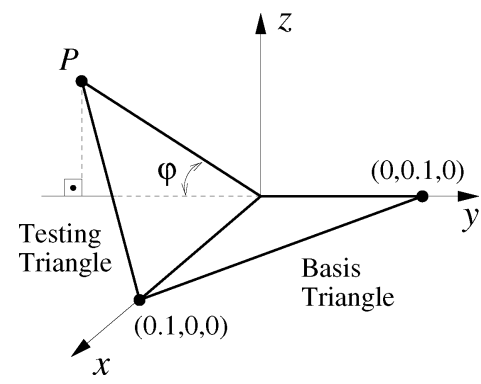

Fig. 2. A specific example of two touching triangles, whose mutual interaction is singular.

where $R_{0}$ is the distance between the the observation point and the common edge. The integrals in the second part of (15) can be evaluated analytically as

$$
\begin{aligned}
\int_{S_{m}} d \boldsymbol{r} 2 \ln \left(R_{0}\right)= & 2 A_{m}\left[\ln \left(h_{m}\right)-\frac{3}{2}\right] \\
\int_{S_{m}} d \boldsymbol{r} x 2 \ln \left(R_{0}\right)= & x_{0} A_{m}\left[\frac{2}{3} \ln \left(h_{m}\right)-\frac{8}{9}\right] \\
& +\left(l_{0}-x_{0}\right) A_{m}\left[\frac{1}{3} \ln \left(h_{m}\right)-\frac{11}{18}\right] \\
\int_{S_{m}} d \boldsymbol{r} x^{2} 2 \ln \left(R_{0}\right)= & x_{0}^{2} A_{m}\left[\frac{1}{2} \ln \left(h_{m}\right)-\frac{5}{8}\right] \\
& +\left(l_{0}-x_{0}\right)^{2} A_{m}\left[\frac{1}{6} \ln \left(h_{m}\right)-\frac{25}{72}\right] \\
& +x_{0}\left(l_{0}-x_{0}\right) A_{m}\left[\frac{1}{2} \ln \left(h_{m}\right)+\frac{7}{8}\right]
\end{aligned}
$$

where $A_{m}$ and $h_{m}$ are the area and height of the testing triangle, respectively, while $l_{0}$ is the length of the common edge and $x_{0}$ is the $x$ coordinate of the vertex across from the common edge. The integrals in the first part of (15) can be evaluated numerically as usual since the integrands of the outer integrals are not singular and have finite limits on the common edge.

\section{RESULTS}

The improvement obtained by the proposed singularity-extraction technique will be demonstrated by computing the nearneighbor interaction between the basis and testing triangles depicted in Fig. 2, where the coordinate values are specified in terms of the wavelength $(\lambda)$. The testing triangle makes an angle $\varphi$ with the $x-y$ plane so that the coordinates of the point $P$ (in $\lambda)$ are given by

$$
P=(0,-0.1 \cos \varphi, 0.1 \sin \varphi) .
$$

By considering a half RWG function as $\boldsymbol{t}_{n}(\boldsymbol{r})$ on the testing triangle and a half RWG function as $\boldsymbol{b}_{n}(\boldsymbol{r})$ on the basis triangle, both associated with the common edge $n$, the partial matrix element $Z_{n n(1)}^{M}$ is computed by employing increasing orders of numerical quadrature on the testing integral [11]. Since the singularity-extraction improvements outlined in Section III are related to the imaginary part of $Z_{n n(1)}^{M}$, Figs. 3 and 4 show the computed value of $\operatorname{Im}\left\{Z_{n n(1)}^{M}\right\}$ for $\varphi=30^{\circ}$ and $\varphi=60^{\circ}$, respectively, with respect to the number of quadrature points [11] employed for the outer integral on the testing triangle. Since the inner integrals are computed with an error criterion of $1 \%$, the

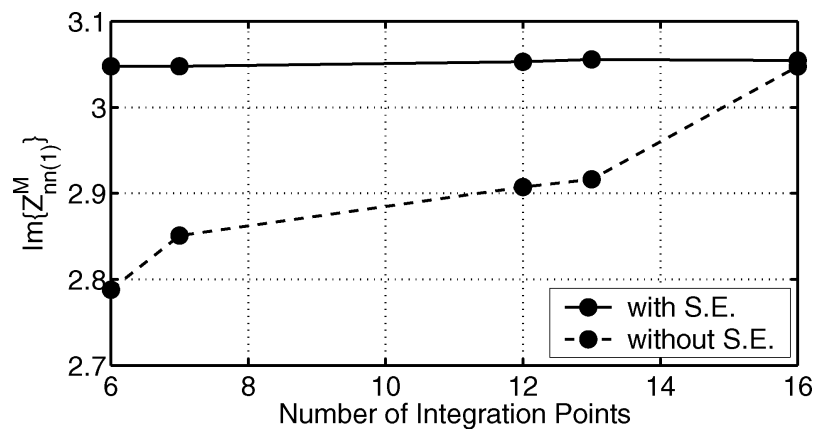

Fig. 3. Values of $\operatorname{Im}\left\{Z_{n n(1)}^{M}\right\}$ computed with and without the singularity extraction for the configuration of Fig. 2 and $\varphi=30^{\circ}$.

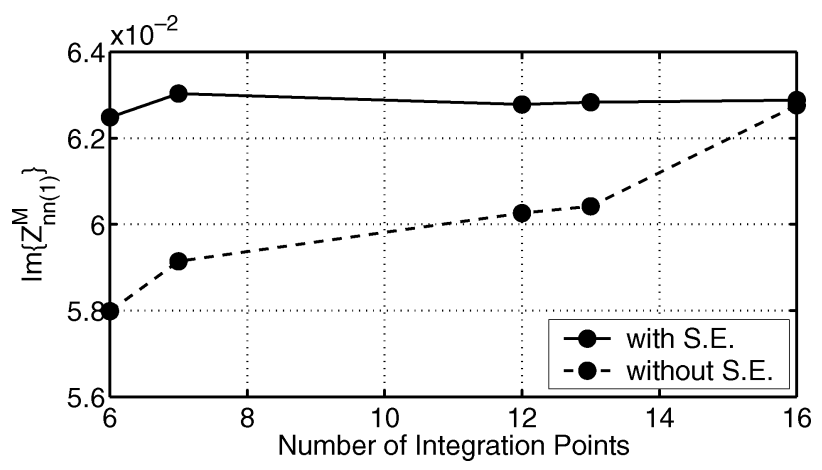

Fig. 4. Values of $\operatorname{Im}\left\{Z_{n n(1)}^{M}\right\}$ computed with and without the singularity extraction for the configuration of Fig. 2 and $\varphi=60^{\circ}$.

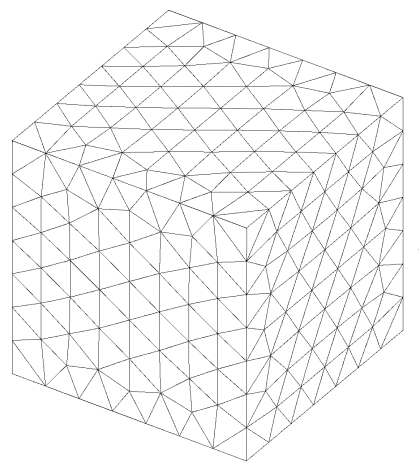

(a)

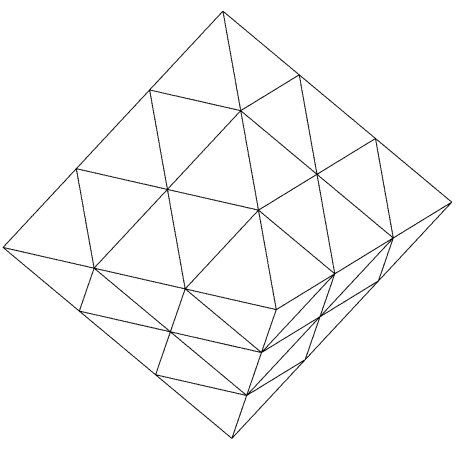

(b)
Fig. 5. (a) Perfectly conducting cube with edges of $1-\lambda$ and mesh size of $\lambda / 10$. (b) Perfectly conducting small octahedron with edges of $0.07-\lambda$ and mesh size of $\lambda / 43$.

errors in $\operatorname{Im}\left\{Z_{n n(1)}^{M}\right\}$ are mostly due to the outer integrals. Both Figs. 3 and 4 establish that the singularity extraction in the outer integrals significantly improves the convergence with respect to the number of integration points and the matrix elements are calculated more accurately with fewer integration points.

Finally, two perfectly conducting geometries are displayed in Fig. 5. To demonstrate the effect of the singularity extraction on the results of the entire problem, Figs. 6 and 7 present the monostatic radar cross section (RCS) values for the two perfectly conducting geometries displayed in Fig. 5. The first geometry is a cube with edges of $1 \lambda$ and mesh size of about $0.1 \lambda$, while the second geometry is a small octahedron with edges of $0.07 \lambda$ and mesh size of about $0.023 \lambda$. In both cases, the excitation is defined as a plane wave illuminating the top of the geometry, and 


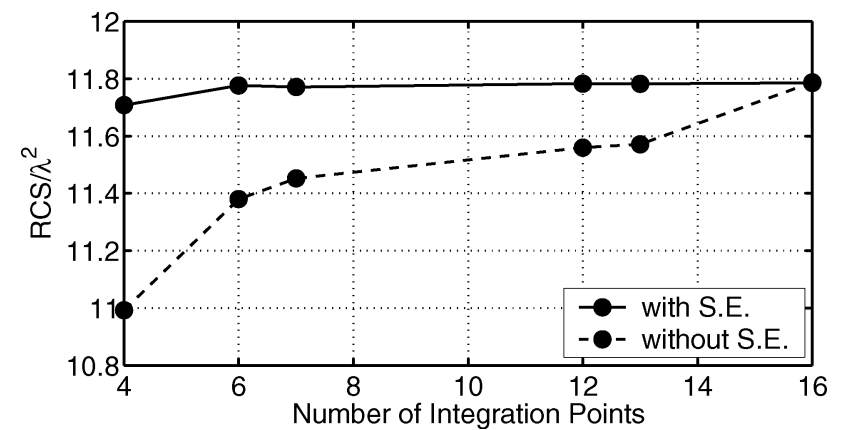

Fig. 6. Monostatic RCS values for the cube in Fig. 5(a) computed with and without the singularity extraction.

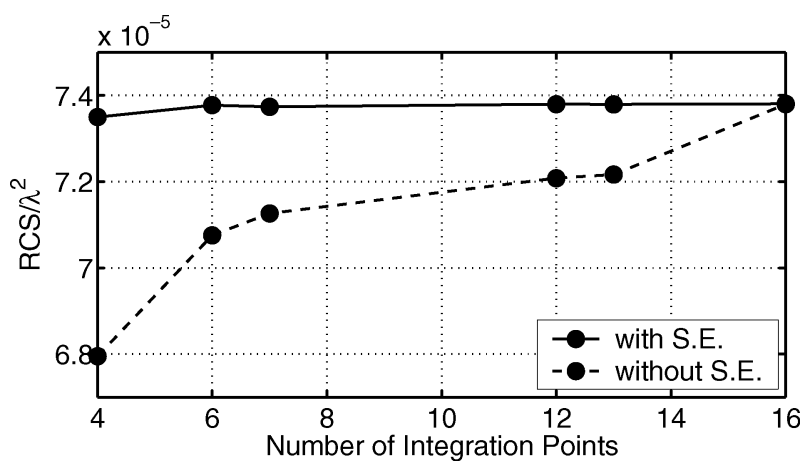

Fig. 7. Monostatic RCS values for the octahedron in Fig. 5(b) computed with and without the singularity extraction.

the backscattering problem is solved. Monostatic RCS values presented in Figs. 6 and 7 confirm the findings already inferred from Figs. 3 and 4, namely, that the singularity extraction improves the accuracy of the results even if the outer testing integrals are computed with a few sampling points.

\section{CONCLUSION}

In this letter, we first show that various formulations of the MFIE and the EFIE are possible and that they impose different restrictions on the BTFs. In principle, the EFIE is considered to be more singular than the MFIE due to the higher-order derivative in the EFIE kernel. Nevertheless, we indicate that the most commonly employed formulation of the MFIE (5) has a more singular kernel than the popularly preferred formulation of the EFIE (9). The highly singular inner integral of (5) can be extracted and analytically evaluated, only to obtain a logarithmic function, which in turn renders the outer integral singular.

We propose a novel singularity-extraction technique for the computation of the singular outer integrals of the MFIE in an accurate and efficient manner. Benefits of the technique are demonstrated at the levels of both individual matrix elements and overall scattering results. It is established that the proposed singularity-extraction technique improves the accuracy at both levels without having to increase the number of quadrature points. Another interpretation of the same results suggests that the technique improves the efficiency of the numerical computation of the MFIE integrals without sacrificing accuracy.

\section{REFERENCES}

[1] Ö. Ergül and L. Gürel, "Investigation of the inaccuracy of the MFIE discretized with the RWG basis functions," in Proc. 2004 IEEE AP-S Int. Symp. and URSI Radio Science Meeting, Monterey, CA, pp. 3393-3396.

[2] R. D. Graglia, "On the numerical integration of the linear shape functions times the 3-D Green's function or its gradient on a plane triangle," IEEE Trans. Antennas Propag., vol. 41, no. 10, pp. 1448-1455, Oct. 1993.

[3] R. E. Hodges and Y. Rahmat-Samii, "The evaluation of MFIE integrals with the use of vector triangle basis functions," Microw. Opt. Technol. Lett., vol. 14, no. 1, pp. 9-14, Jan. 1997.

[4] S. M. Rao, D. R. Wilton, and A. W. Glisson, "Electromagnetic scattering by surfaces of arbitrary shape," IEEE Trans. Antennas Propag., vol. AP-30, no. 3, pp. 409-418, May 1982.

[5] Ö. Ergül and L. Gürel, "Improving the accuracy of the MFIE with the choice of basis functions," in Proc. 2004 IEEE AP-S Int. Symp. and URSI Radio Science Meeting, Monterey, CA, pp. 3389-3392.

[6] A. J. Poggio and E. K. Miller, "Integral equation solutions of three-dimensional scattering problems," in Computer Techniques Electromagnetics, R. Mittra, Ed. Oxford, U.K.: Permagon, 1973, ch. 4.

[7] J. R. Mautz and R. F. Harrington, "H-field, E-field, and combined field solutions for conducting bodies of revolution," $A E \ddot{U}$, vol. 32, no. 4, pp. 157-164, Apr. 1978.

[8] N. Morita, N. Kumagai, and J. R. Mautz, Integral Equation Methods for Electromagnetics. Norwood, MA: Artech House, 1990.

[9] A. F. Peterson, S. L. Ray, and R. Mittra, Computational Methods for Electromagnetics. Piscataway, NJ: IEEE Press, 1998.

[10] Y. Zhang, T. J. Cui, W. C. Chew, and J.-S. Zhao, "Magnetic field integral equation at very low frequencies," IEEE Trans. Antennas Propag., vol. 51, no. 8, pp. 1864-1871, Aug. 2003.

[11] D. A. Dunavant, "High degree efficient symmetrical Gaussian quadrature rules for the triangle," Int. J. Numer. Methods Eng., vol. 21, pp. 1129-1148, 1985. 